\title{
Online Optimization for Real-Time Peer-to-Peer Electricity Market Mechanisms
}

\author{
Guo, Zhenwei; Pinson, Pierre; Chen, Shibo; Yang, Qinmin; Yang, Zaiyue
}

Published in:

IEEE Transactions on Smart Grid

Link to article, DOI:

10.1109/TSG.2021.3075707

Publication date:

2021

Document Version

Peer reviewed version

Link back to DTU Orbit

Citation (APA):

Guo, Z., Pinson, P., Chen, S., Yang, Q., \& Yang, Z. (2021). Online Optimization for Real-Time Peer-to-Peer Electricity Market Mechanisms. IEEE Transactions on Smart Grid, 12(5), 4151 - 4163.

https://doi.org/10.1109/TSG.2021.3075707

\section{General rights}

Copyright and moral rights for the publications made accessible in the public portal are retained by the authors and/or other copyright owners and it is a condition of accessing publications that users recognise and abide by the legal requirements associated with these rights.

- Users may download and print one copy of any publication from the public portal for the purpose of private study or research.

- You may not further distribute the material or use it for any profit-making activity or commercial gain

- You may freely distribute the URL identifying the publication in the public portal

If you believe that this document breaches copyright please contact us providing details, and we will remove access to the work immediately and investigate your claim. 


\title{
Online Optimization for Real-Time Peer-to-Peer Electricity Market Mechanisms
}

\author{
Zhenwei Guo, Student Member, IEEE, Pierre Pinson, Fellow, IEEE, Shibo Chen, Member, IEEE, \\ Qinmin Yang, Senior Member, IEEE, and Zaiyue Yang, Member, IEEE
}

\begin{abstract}
Owing to the fast deployment of distributed energy resources (DERs) and the further development of demand-side management, small agents in electricity markets are becoming more proactive. This may boost the development of peer-topeer (P2P) market mechanisms. Meanwhile, since actual load and power generation may substantially deviate from schedules obtained at the day-ahead (forward) market stage, it is needed to rapidly reschedule the trades among agents to maintain power balance through a real-time market mechanism, also in a P2P framework. However, it is technically challenging to develop and operate such P2P market mechanisms in real-time, since they most often involve a heavy computational burden (e.g., based on iterative distributed optimization approaches), while real-time trading demands fast calculation. Our core contribution is hence to describe and analyze a novel online optimization framework to enable the real-time P2P market. It relies on online social welfare maximization using a novel online consensus alternating direction method of multipliers (OC-ADMM) algorithm. The computational complexity is then heavily reduced since only one iteration is performed for each agent at every time step in order to satisfy real-time requirements. We derive a sublinear nonstationary regret upper bound for our algorithm, which implies that social welfare will be maximized in the long run. Simulations based on a number of case studies show that our algorithm has good convergence performance, tracking ability, and high computational efficiency.
\end{abstract}

Index Terms-Real-time P2P market, online consensus ADMM, primal-dual alternate update, non-stationary regret.

\section{NOMENCLATURE}

Functions

$\tilde{R}(\cdot) \quad$ Non-stationary regret.

$C(\cdot) \quad$ Production cost or utility function.

$R(\cdot) \quad$ Stationary regret.

Numbers and Indexes

$k \quad$ Index for iterations.

$l \quad$ Index for iterations.

Manuscript received July 1, 2020; revised November 8, 2020 and February 14, 2021; accepted April 6, 2021. This work was supported in part by National Natural Science Foundation of China $(61873118,61903179)$, in part by the Science, Technology and Innovation Commission of Shenzhen Municipality under Grant RCBS20200714114918137, in part by Zhejiang Provincial Nature Science Foundation of China under Grant LZ21F030004, and in part by Key-Area Research and Development Program of Guangdong Province (No. 2018B010107002). Paper no. TSG-01004-2020. (Corresponding author: Zaiyue Yang.)

Zhenwei Guo and Qinmin Yang are with the State Key Laboratory of Industrial Control Technology, Zhejiang University, Hangzhou 310027, China (e-mail: zhenweiguo0724@163.com, qmyang@zju.edu.cn).

Shibo Chen and Zaiyue Yang are with Department of Mechanical and Energy Engineering, Southern University of Science and Technology, Shenzhen 518055, China (e-mail: chensb@ sustech.edu.cn, yangzy3@ sustc.edu.cn)

Pierre Pinson is with the Center for Electric Power and Energy, Danmarks Tekniske Universitet, Denmark, e-mail: ppin@dtu.dk.
$N \quad$ Cardinal number of agents.

$n, m \quad$ Indices for agents.

$N_{\omega} \quad$ Cardinal number of neighboring agents.

$T \quad$ Cardinal number of time steps.

$t \quad$ Indices for time steps.

Parameters

$\chi^{c} \quad$ Nomalized cost deviation.

$\epsilon \quad$ Allowed maximal violation of constraint (1).

$\rho, \eta \quad$ Penalty factors.

$\underline{E}, \bar{E} \quad$ Boundaries of power.

$\varepsilon \quad$ Allowed maximal violation of trade between agents.

$a, b \quad$ Coefficients of the quadractic fucntions.

$G, D, P, L, \Lambda$ Positive upper bounded constants in assumptions. $P^{\min }, P^{\max }$ The line thermal limits.

$V \quad$ Path variation.

$Y \quad$ The susceptance of the line.

\section{Sets and Vectors}

$\lambda \quad$ Vector of whole transaction prices.

$\boldsymbol{\theta} \quad$ Vector of all voltage angles of buses.

E Vector of whole transaction quantites.

$\mathbf{P} \quad$ Vector of all power flows in lines.

$\mathcal{L} \quad$ Set of lines.

$\mathcal{N} \quad$ Set of buses.

$\Omega \quad$ Set of agents.

$\omega \quad$ Set of neighboring agents.

$\Omega_{p}, \Omega_{c}, \Omega_{p s}$ Set of producers, consumers and prosumers.

Variables

$\hat{E} \quad$ The final trade quantity after projection.

$\hat{E}^{\text {proj }}$ The projected total power.

$\lambda \quad$ Transaction price.

$\theta \quad$ The voltage angle at but.

$\tilde{\delta} \quad$ Average value of the dual variables $\delta$ between neighboring agents.

$\tilde{\lambda} \quad$ Average value of the prices between neighboring agents.

$\tilde{F} \quad$ Average value of the consensus variable between neighboring agents.

$\underline{\delta}, \bar{\delta} \quad$ Corresponding dual variables for constraint (3).

$\mu, \bar{\mu} \quad$ Corresponding dual variables for constraint (2).

$\bar{E} \quad$ Power injection or transaction quantity.

$E^{*} \quad$ Optimal power injection or transaction quantity.

$F \quad$ Consensus variable of trades.

$P \quad$ The real power flow in the line.

\section{INTRODUCTION}

The ever-increasing distributed energy resources (DERs) and demand response (DR) are changing the way of power 
system operation. Participants are becoming more proactive in the market, who prefer determining the trading outcomes by themselves. Therefore, electricity markets are evolving towards more decentralized mechanisms. However, current electricity markets still perform resource allocation and pricing based on the conventional hierarchical and top-down approach [1], which makes participants behave as passive receivers. Recently, a novel design of electricity markets has emerged: these so-called peer-to-peer $(\mathrm{P} 2 \mathrm{P})$ electricity markets rely on multi-bilateral trades between each pair of participants [2][11]. Employing a P2P market mechanism can yield plenty of advantages, e.g., empowerment of participants, increasing the resilience of power system and protection of privacy [2], [5]. Existing works about P2P markets mainly focus on the following issues: reallocation of the costs [3], product differences [4], [9], dispatch fairness [8], flexibility of battery [10], [11], communication properties [6] and costs [7]. Technically, different decentralized methods are devised to realize market mechanism, for instance, primal-dual gradient [4], relaxed consensus+innovation [6], [9], standard alternating direction method of multipliers (ADMM) [8], stochastic programming [11] and consensus ADMM [3], [7].

Meanwhile, in actual operation, due to changes in the weather, potential power system accidents, renewable power generation uncertainty, demand-side load variations, and other contingencies, the actual load and power generation may have a large deviation from the schedule obtained at the day-ahead (or more generally forward) market stage - power balance hence ought to be restored. Thus, it is required to rapidly reschedule the trades among market participants to keep power balance in response to these changes - hence calling for realtime markets (RTMs) [12]-[15]. The main function of RTMs is to build an efficient transaction platform for small DERs and DR to actively provide balancing services during power system operations [16]. Small DERs and DR have the potentials to provide faster balancing services than conventional generators, which usually provide balancing services within 15-20 min. Compared with day-ahead and intraday markets, the time-scale for the real-time market for settlement before operation is usually $5 \mathrm{~min}$ or even shorter. Therefore, the main operational requirement for agents to participate in the RTMs is fast enough to provide balancing services to continuously keep supply-demand balance in response to the changes, so that the stability and reliability of the power system can be improved. Thus, the fast calculation is essential to a real-time market. However, a $\mathrm{P} 2 \mathrm{P}$ market mechanism requires a very large amount of information to be exchanged, much greater than that required for a centralized market [6], [7], [17]. In a realtime market context, such exchanges run a risk of not having enough time to succeed if the deadline is reached before the end of the negotiation process. Eventually, the main challenge for a $\mathrm{P} 2 \mathrm{P}$ real-time electricity market is how to lower the computational complexity of the $\mathrm{P} 2 \mathrm{P}$ mechanism so that it can be deployed in a real-time architecture.

Note that some works already considered P2P mechanisms for real-time electricity markets [18]-[21]. For instance, [18] proposed bilateral contract networks as a new market design for $\mathrm{P} 2 \mathrm{P}$ energy trading in real-time markets, but it did not consider how one could take advantage of previous negotiations to make the current negotiation faster (also knowing that the agents update their information and preferences). Ref. [19] proposed a P2P local electricity market model incorporating both energy trading and uncertainty trading simultaneously, also based on a contract matching mechanism, though at the day-ahead stage only. The other two works [20], [21] employed blockchain-based approaches within a P2P real-time market, but from the communication network level, prices and payments are still centrally determined by the central coordinator, and not via a P2P structure. However, these works ignored the computational efficiency trouble of the P2P realtime market.

As it may be too expensive to optimally solve a P2P market at each and every time step, one needs to think of appropriate and computationally cheaper approaches. Compared with previous related works [3], [5], [9], [22], [23], we innovatively design an online optimization framework to enable the realtime P2P market. Online optimization [24]-[26] deals with optimization problems that have no or incomplete information of the future. These kinds of problems are denoted as online problems and seen as opposed to the classical optimization problems where complete information is obtained. The decisions can only be made in an online manner based on piece-by-piece input information since the environment is assumed to be uncertain and changing overtimes. In general, the output of an online algorithm is compared to the result of a corresponding offline algorithm which is optimal and knows the entire information in advance. Despite many large-scale applications of online optimization, such as network resource allocation [27], [28], demand response [29], [30], and energy management [31], our work is the first application for P2P electricity market.

To be specific, instead of optimally solving a complete classical P2P market at each and every time, we concentrate on an alternative paradigm aiming to maximize the social welfare in the long run, using a novel online consensus alternating direction method of multipliers (OC-ADMM) algorithm - this is computationally lighter and more tractable. The number of operations and communications among agents can be heavily reduced, and the complexity of our approach is less than other approaches for a given setup since the OC-ADMM algorithm only performs one iteration for every single agent at every time step. For example, [22] proposed a P2P joint energy and reserve market, using a consensus-based ADMM algorithm, which may cost over 100 iterations to reach the optimal solution. It is not applicable in the real-time market since computational time may exceed the requirement of the real-time market (5 min). Another work [6] investigated the convergence performance of different distributed algorithms to enable the $\mathrm{P} 2 \mathrm{P}$ market mechanism, which all required hundreds of iterations to converge, and will increase with the number of agents.

Moreover, the proposed algorithm can be further improved in three aspects: 1) local optimization problems can be reduced to analytical formulations by using a primal-dual variable alternate update process, which can greatly improve the computational speed; 2) the transaction power between each pair 
of two agents can be balanced using a projection-based power update process. 3) the network constraints can be satisfied by adding a system operator ( $\mathrm{SO}$ ) who behaves as a single agent and helps to complete the calculation of power flows and voltage angles. Finally, for our proposed online algorithm, the sublinear regret upper bound is also proved, which implies that social welfare is maximized in the long run. In simulations, a simple case is given to illustrate the trading process. Then, real wind power data is employed to show the convergence performance and tracking ability of our algorithm. Finally, the comparison between our algorithm and other methods demonstrates our high computational speed. Our contributions mainly lie as follows.

- We innovatively propose an online optimization framework to enable the P2P real-time electricity market. The computational complexity of the $\mathrm{P} 2 \mathrm{P}$ mechanism can be highly reduced to be within the operational requirement of real-time mechanisms since the proposed algorithm OC-ADMM only performs one iteration for each agent at every time step.

- We further improve the online algorithm by devising some techniques: a primal-dual variable alternate update process to improve the computational speed, a projectionbased power update process to guarantee the power balance, and adding a SO who behaves as a single agent to complete the calculation of power flows and voltage angles.

- The sublinear non-stationary regret upper bound for our online algorithm is proved, which implies that social welfare will be maximized in the long run on time average.

The rest of the paper is organized as follows: Section II presents the real-time P2P electricity market model. Section III proposes the OC-ADMM algorithm and improvements for it, followed by the regret and market properties analysis in Section IV. Numerical results and comparisons are presented in Section V. Finally, conclusions are drawn in Section VI.

\section{REAL-Time PEeR-TO-PEer Electricity MARKeT}

We consider a real-time P2P electricity market with a set $\Omega$ of $N$ dynamic and proactive agents who can be traditional generators, consumers with flexible loads, and renewable generators (wind and PV) over a time horizon $T$. The term "dynamic" means the characteristics of agents, e.g., the cost or utility function coefficients, energy demand, and renewable power generation are changing with time. The term "proactive" means the agents are very willing to participate in the market to trade and negotiate with others to determine electricity prices and quantities. As it is classically done, agents are supposed rational as in [32], i.e. always objectively taking the most beneficial decisions, and non-strategic, i.e., not anticipating actions and reactions of other agents.

\section{A. Peer-to-Peer Trading}

A P2P mechanism for electricity markets is much more decentralized compared with existing centralized markets, which consists of synchronous negotiations over the price and quantity between each pair of two agents. To model the trading process, the net power injection $E_{n, t}$ of each agent $n \in \Omega$ at time step $t$ is split into a sum of bilaterally transaction quantities with a set of neighboring agents $m \in \omega_{n}$ as

$$
E_{n, t}=\sum_{m \in \omega_{n}} E_{n m, t}, \quad \forall n \in \Omega, t=1, \ldots, T .
$$

A positive value of $E_{n m, t}$ corresponds to a sale/production and a negative value to a purchase/consumption. To lighten notations, $\mathbf{E}_{n, t}=\left\{E_{n 1, t}, \ldots, E_{n m, t}\right\}$ is used to represent the whole transaction quantities of agent $n$ at time step $t$. The power set-point of an agent $n$ is constrained as

$$
\underline{E}_{n, t} \leq E_{n, t} \leq \bar{E}_{n, t}, \quad \forall n \in \Omega, t=1, \ldots, T .
$$

Here, for renewable generators, the upper bound $\bar{E}_{n, t}$ is set to the smaller value of actual power generation and maximal capacity. Each agent can be a producer $\left(0 \leq \underline{E}_{n, t} \leq \bar{E}_{n, t}\right)$, a consumer $\left(\underline{E}_{n, t} \leq \bar{E}_{n, t} \leq 0\right)$ or a prosumer $\left(\underline{E}_{n, t} \leq 0 \leq\right.$ $\left.\bar{E}_{n, t}\right)$. Hence, $E_{n m, t}$ are constrained as

$\left\{\begin{array}{l}E_{n, t} \geq E_{n m, t} \geq 0, \quad \forall(n, m) \in\left(\Omega_{p}, \omega_{n}\right), t=1, \ldots, T \\ E_{n, t} \leq E_{n m, t} \leq 0, \quad \forall(n, m) \in\left(\Omega_{c}, \omega_{n}\right), t=1, \ldots, T, \\ \underline{E}_{n, t} \leq E_{n m, t} \leq \bar{E}_{n, t}, \forall(n, m) \in\left(\Omega_{p s}, \omega_{n}\right), t=1, \ldots, T,\end{array}\right.$

where $\Omega_{p}, \Omega_{c}$ and $\Omega_{p s}$ are the sets of producers, consumers and prosumers, respectively. Finally, the market equilibrium between production and consumption is represented by a set of reciprocity balance constraints defined as

$$
E_{n m, t}+E_{m n, t}=0, \quad \forall(n, m) \in\left(\Omega, \omega_{n}\right), t=1, \ldots, T .
$$

For the aim of better formulation, the generator production cost and consumer utility $C_{n, t}\left(E_{n, t}\right)$ (positive for producers while negative for consumers) are assumed to be quadratic functions [3], [9], though our mechanism is still applicable for any general convex functions.

$$
C_{n, t}\left(E_{n, t}\right)=\frac{1}{2} a_{n, t} E_{n, t}^{2}+b_{n, t} E_{n, t} .
$$

Remark 1. The time-coupling constraints, e.g., the ramp rate limits for conventional generators and total demand requirement for consumers, can also be included. To be specific, since the market is running in an online fashion, the agents do not know future information, they can only make decisions based on current or past information. Then, the time-coupling constraints, e.g., ramping limit or total demand requirement, at time step $t$ for agent $n$ can be formulated as

$$
\begin{aligned}
& E_{n, t}-E_{n, t-1} \leq \Delta E_{n}, \quad \forall n \in \Omega_{p}, t=1, \ldots, T, \\
& E_{n, t-1}-E_{n, t} \leq \Delta E_{n}, \quad \forall n \in \Omega_{p}, t=1, \ldots, T, \\
& E_{n, t}+\sum_{t=1}^{l-1} E_{n, l} \leq D_{n, t}, \quad \forall n \in \Omega_{c}, t=1, \ldots, T,
\end{aligned}
$$

where $E_{n, t-1}$ is the decision variable determined at last time step, $\Delta E_{n}$ is the ramp limit for conventional generator $n ; D_{n, t}$ is the minimal total required demand for consumer $n$ in the first t time steps. (6a) and (6b) enforce the ramping up/down constraints for conventional generators, and (6c) implies that the total required demand is bounded (remember $E_{n, t}$ is 
negative for consumer). Above constraints can be reformulated as

$$
\begin{aligned}
& E_{n, t-1}-\Delta E_{n} \leq E_{n, t} \leq E_{n, t-1}+\Delta E_{n}, \quad \forall n \in \Omega_{p}, \\
& E_{n, t} \leq D_{n, t}-\sum_{t=1}^{l-1} E_{n, l}, \quad \forall n \in \Omega_{c} .
\end{aligned}
$$

It is noted that above time-coupling constraints for producers and consumers can be combined with the power level constraints (2) as

$$
\begin{aligned}
& \max \left\{E_{n, t-1}-\Delta E_{n}, \underline{E}_{n, t}\right\} \leq E_{n, t} \leq \\
& \min \left\{E_{n, t-1}+\Delta E_{n}, \bar{E}_{n, t}\right\}, \quad \forall n \in \Omega_{p} \\
& \underline{E}_{n, t} \leq E_{n, t} \leq \min \left\{D_{n, t}-\sum_{t=1}^{l-1} E_{n, l}, \bar{E}_{n, t}\right\}, \quad \forall n \in \Omega_{c} .
\end{aligned}
$$

Therefore, including the time-coupling constraints will not break the model, and the problem is still solvable by using our algorithm.

\section{B. Social Welfare Maximization Problem}

Since agents do not know the information from the future, they can only make decisions based on current updated and past information. Thus, the market has to be running step by step instead of solving the problem for all $T$. To be more specific, at the beginning of the time step $t$, each agent will be aware of the current updated information, including cost or utility function coefficients, power bounds, demand bounds, and renewable generation. After obtaining these information, the agents will make decisions during the time step $t$. All agents negotiate with each other to reach a consensus on their transaction prices and quantities while maximizing social welfare. Mathematically speaking, this is seen as a minimization problem formulated as

$$
\begin{aligned}
\min _{\left\{E_{n \in \Omega}\right\}} & \sum_{t=1}^{T}\left(\sum_{n \in \Omega} C_{n, t}\left(E_{n, t}\right)\right) \\
\text { s.t. } & (1)-(4) .
\end{aligned}
$$

If the problem (9) is solved exactly at every time step in a decentralized manner, a double loop algorithm is needed where the function $C_{n, t}$ changes in the outer loop, while the inner loop runs iteratively until the balance constraint (4) is satisfied. However, in a P2P market, the number of communication times will increase with the square of the number of agents [7]. Thus, for the problem (9), if we want to obtain the optimal solution at each time step, all agents have to complete many iterations and cause very heavy communication and computational burden. Thus, one needs to think of appropriate and computationally cheaper approaches. To this end, we propose an online optimization framework for the real-time $\mathrm{P} 2 \mathrm{P}$ market, which is more practicable and applicable.

\section{Real-Time P2P Electricity Market Mechanism}

Since the market is running in an online manner, problem (9) is decomposed into each single time step. The social welfare maximization problem (written in a cost-minimization form) for the time step $t$ is

$$
\begin{aligned}
\min _{\left\{\mathbf{E}_{n \in \Omega}\right\}} & \sum_{n \in \Omega}\left(C_{n, t}\left(E_{n}\right)+\sum_{m \in \omega_{n}} \frac{\eta}{2}\left(E_{n m, t-1}-E_{n m}\right)^{2}\right) \\
\text { s.t. } & (1)-(4),
\end{aligned}
$$

where $\eta$ is the penalty factor and the term $\frac{\eta}{2}\left(E_{n m, t-1}-E_{n m}\right)^{2}$ is appended to make the results close to previous value $E_{n m, t-1}$ in order to speed up the convergence process [33], [34].

\section{A. Online Consensus ADMM}

Plain ADMM is an algorithm that blends the decomposability of dual ascent with the superior convergence properties of the method of multipliers, but there is a central coordinator for updating the dual variable [35]. Thus, the plain ADMM is suitable for a distributed market where there is a market operator to help to decide the price. To get rid of the central coordinator, [35] proposed the consensus ADMM method. In this method, all agents can reach a consensus value by only communicating with neighboring agents. Then, [3] proposed a decentralized P2P market based on the consensus ADMM, where each pair of agents will reach a consensus value of the trade quantities. For the plain ADMM, the objective functions are fixed and do not change with time. However, in some practical applications, objection functions usually change with time. To improve the practicability of ADMM methods, [33] proposed the online ADMM, which is an efficient technique that combines plain ADMM with online learning theory. Compared with the plain ADMM, objective functions are changing with time, and the target is to minimize the objective value over a long period of time. Combining above approaches, we propose the novel OC-ADMM algorithm to implement the real-time P2P market, which produces the following updates:

- Power Updates: Each agent $n$ updates their transaction quantities with neighboring agents by solving individual optimization problem with constraints (1)-(3) as below:

$$
\begin{aligned}
& \mathbf{E}_{n, t}=\underset{\mathbf{E}_{n}}{\operatorname{argmin}} C_{n, t}\left(E_{n}\right)+\sum_{m \in \omega_{n}} \lambda_{n m, t-1}\left(F_{n m, t-1}-E_{n m}\right) \\
& +\frac{\rho}{2}\left(F_{n m, t-1}-E_{n m}\right)^{2}+\frac{\eta}{2}\left(E_{n m}-E_{n m, t-1}\right)^{2},
\end{aligned}
$$

where $\rho$ is the penalty factor and $\lambda_{n m, t-1}$ is the dual variable of the reciprocity constraint (4), which also defines the price for transaction quantity $E_{n m, t-1} \cdot \boldsymbol{\lambda}_{n, t-1}=$ $\left\{\lambda_{n 1, t-1}, \ldots, \lambda_{n m, t-1}\right\}$ is used to represent whole transaction prices of agent $n$ to neighboring agents $m \in \omega_{n}$ at time step $t-1 . F_{n m, t-1}$ is the consensus variable defined as $\frac{E_{n m, t-1}-E_{m n, t-1}}{2}$ and $F_{n, t-1}=\sum_{m \in \omega_{n}} F_{n m, t-1}$. Then, each agent broadcasts $\mathbf{E}_{n, t}$ to neighboring agents.

- Price Updates All agents update their transaction prices to neighboring agents $m \in \omega_{n}$ as:

$$
\lambda_{n m, t}=\lambda_{n m, t-1}-\rho\left(E_{n m, t}+E_{m n, t}\right) / 2 .
$$

The OC-ADMM algorithm is much more efficient since each agent only needs to complete one iteration at each 
time step. However, the algorithm can be further improved. First, the update (11) is still an optimization problem, which can speed up by transforming into analytical expressions; second, since we only run one iteration at each time step, the power balance between two agents may not be balanced, i.e., $E_{n m, t}+E_{m n, t} \neq 0$, and a market mechanism needs to keep the trading balance; third, the network constraints are important in power systems and should be considered in the market.

\section{B. Improvements for $O C-A D M M$}

In this section, we make three further improvements, which are 1) a primal-dual variables alternate update process to increase the computational speed, 2) a projection-based power update process to guarantee the power balance, and 3) adding a SO who behaves as a single agent to complete the calculation of power flows and voltage angles. The procedures of the improved OC-ADMM are summarized in Algorithm 1.

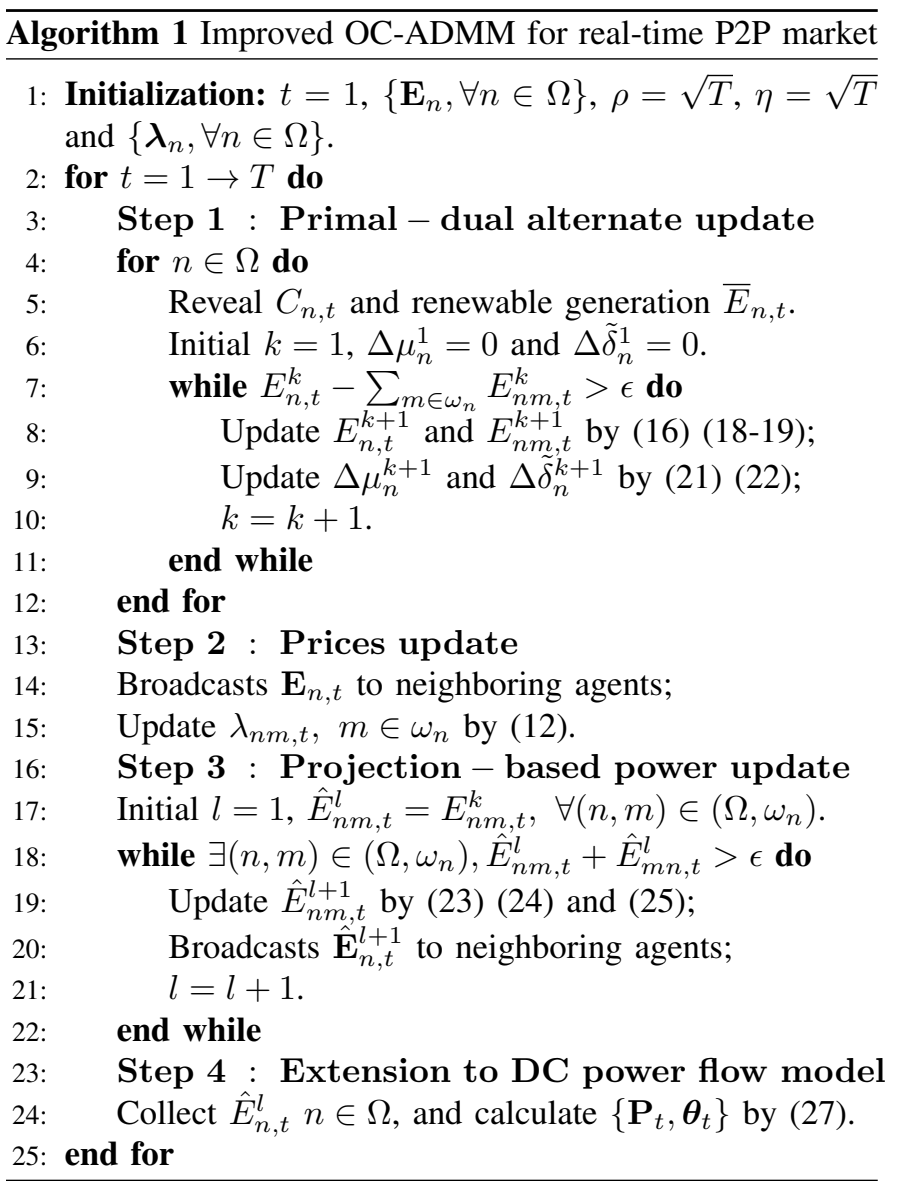

1) Primal-Dual Variables Alternate Update: Since the individual problem (11) is a convex optimization problem, we can obtain the optimal solution by equivalently solving its Karush-Kuhn-Tucker (KKT) conditions [36], which is a nonlinear problem with primal variables $\left\{E_{n, t}^{k+1}, \mathbf{E}_{n, t}^{k+1}\right\}$ and dual variables $\left\{\underline{\mu}_{n}, \bar{\mu}_{n}\right\},\left\{\underline{\delta}_{n m}, \bar{\delta}_{n m}\right\}$ for constraints (2) and (3). This problem can be solved in a primal-dual variables alternate update manner. Since update processes are all analytical expressions, the computational speed can be greatly increased. In this process, iterations are indexed with $k$.
Primal Updates: Relaxing the constraint (1) and based on the KKT conditions of (11) for a trade with $m \in \omega_{n}$, we have

$$
\begin{aligned}
& \partial C_{n, t}\left(E_{n, t}\right)-\lambda_{n m, t-1}+\rho\left(E_{n m, t}-F_{n m, t-1}\right) \\
& +\eta\left(E_{n m, t}-E_{n m, t-1}\right)-\underline{\mu}_{n}+\bar{\mu}_{n}-\underline{\delta}_{n m}+\bar{\delta}_{n m}=0 .
\end{aligned}
$$

Summing up (13) for all $m \in \omega_{n}$, we have

$$
\begin{aligned}
& N_{\omega_{n}}\left(a_{n, t} E_{n, t}+b_{n, t}\right) \\
& =\sum_{m \in \omega_{n}} \lambda_{n m, t-1}-(\rho+\eta) E_{n, t}+\rho F_{n, t-1}+\eta E_{n, t-1} \\
& \quad+N_{\omega_{n}}\left(\underline{\mu}_{n}-\bar{\mu}_{n}\right)+\sum_{m \in \omega_{n}}\left(\underline{\delta}_{n m}-\bar{\delta}_{n m}\right) .
\end{aligned}
$$

For simplicity, $\mu-\bar{\mu}$ and $\underline{\delta}-\bar{\delta}$ are denoted by $\Delta \mu$ and $\Delta \delta$, respectively. Given the results from last iteration $k, E_{n, t}^{k+1}$ is updated as

$$
E_{n, t}^{k+1}=\mathcal{V}_{n, t}+\frac{\Delta \mu_{n}^{k}+\Delta \tilde{\delta}_{n}^{k}}{a_{n, t}+\frac{\rho+\eta}{N_{\omega_{n}}}},
$$

where $\mathcal{V}_{n, t}=\left(\tilde{\lambda}_{n, t-1}+\rho \tilde{F}_{n, t-1}+\eta \tilde{E}_{n, t-1}-b_{n, t}\right) /\left(a_{n, t}+\right.$ $\left.\frac{\rho+\eta}{N_{\omega_{n}}}\right), \tilde{\lambda}_{n, t-1}, \tilde{F}_{n, t-1}, \tilde{E}_{n, t-1}$ and $\Delta \tilde{\delta}_{n}^{k}$ are the average values. Based on the complementary condition, $E_{n, t}^{k+1}$ can be obtained in another equivalent form as

$$
E_{n, t}^{k+1}=\max \left\{\min \left\{\mathcal{V}_{n, t}+\frac{\Delta \tilde{\delta}_{n}^{k}}{a_{n, t}+\frac{\rho+\eta}{N_{\omega_{n}}}}, \bar{E}_{n, t}\right\}, \underline{E}_{n, t}\right\} .
$$

According to (13), the trade to $m \in \omega_{n}$ is updated as

$$
E_{n m, t}^{k+1}=\mathcal{W}_{n m, t}+\frac{\Delta \mu_{n}^{k}+\Delta \delta_{n}^{k}-a_{n, t} E_{n, t}^{k+1}}{\rho+\eta},
$$

where $\mathcal{W}_{n m, t}=\left(\lambda_{n m, t-1}+\rho F_{n m, t-1}+\eta E_{n m, t-1}-b_{n, t}\right) /(\rho+$ $\eta)$. Similarly, we can obtain $E_{n m, t}^{k+1}$, for a producer, in another form as

$$
\max \left\{\min \left\{\mathcal{W}_{n m, t}+\frac{\Delta \mu_{n}^{k}-a_{n, t} E_{n, t}^{k+1}}{\rho+\eta}, E_{n, t}^{k+1}\right\}, 0\right\} .
$$

If agent $n$ is a consumer, the $E_{n m, t}^{k+1}$ is updated as

$$
\max \left\{\min \left\{\mathcal{W}_{n m, t}+\frac{\Delta \mu_{n}^{k}-a_{n, t} E_{n, t}^{k+1}}{\rho+\eta}, 0\right\}, E_{n, t}^{k+1}\right\} .
$$

If agent $n$ is a prosumer, the $E_{n m, t}^{k+1}$ is updated as

$$
\max \left\{\min \left\{\mathcal{W}_{n m, t}+\frac{\Delta \mu_{n}^{k}-a_{n, t} E_{n, t}^{k+1}}{\rho+\eta}, \bar{E}_{n, t}\right\}, \underline{E}_{n, t}\right\} .
$$

Dual Updates: From (15), the dual variables $\mu_{n}^{k+1}$ is updated as

$$
\Delta \mu_{n}^{k+1}=\left(a_{n, t}+\frac{\rho+\eta}{N_{\omega_{n}}}\right)\left(E_{n, t}^{k+1}-\mathcal{V}_{n, t}\right)-\Delta \tilde{\delta}_{n}^{k} .
$$

From (17), the average dual variable $\tilde{\delta}_{n}^{k+1}$ is updated as

$$
\Delta \tilde{\delta}_{n}^{k+1}=(\rho+\eta)\left(\tilde{E}_{n, t}^{k+1}-\tilde{\mathcal{W}}_{n, t}\right)-\Delta \mu_{n}^{k+1}+a_{n, t} E_{n, t}^{k+1} .
$$

After updating the dual variables $\left\{\Delta \mu_{n}^{k+1}, \Delta \tilde{\delta}_{n}^{k+1}\right\}$, we go back to update the primal variables $\left\{E_{n, t}^{k+1}, \mathbf{E}_{n, t}^{k+1}\right\}$. This 
process repeats until $\left|E_{n, t}^{k}-\sum_{m \in \omega_{n}} E_{n m, t}^{k}\right|<\epsilon$, where $\epsilon$ is the allowed maximal violation.

In conclusion, instead of solving the optimization problem (11), the primal and dual variables are updated in an alternate iteration process, which is more fast since they are all simple analytical calculations.

Remark 2. The primal-dual variable alternate update process requires the objective functions to be quadratic forms, so that the optimization problems can be reduced to analytical calculations. However, even if the objective functions are general convex forms, the online consensus ADMM is still quite efficient to satisfy the requirement of real-time market mechanisms as shown in the simulations.

2) Projection-based Power Update: In the electricity market, the power balance for trade between agents is necessary. To this end, we design a projection-based power update process to determine the final quantity by projecting the consensus value $F_{n m, t}=\left(E_{n m, t}-E_{m n, t}\right) / 2$ into feasible region (2). In this process, iterations are indexed with $l$.

First, we set the trade $\hat{E}_{n m, t}^{l+1}$ to be the intermediate value of the trade between agents $n$ and $m$ from last iteration as

$$
\hat{E}_{n m, t}^{l+1}=\hat{F}_{n m, t}^{l}=\left(\hat{E}_{n m, t}^{l}-\hat{E}_{m n, t}^{l}\right) / 2 .
$$

Here, the initial value $\hat{E}_{n m, t}^{1}$ is obtained after the primaldual alternate update process, i.e., $\hat{E}_{n m, t+1}^{1}=E_{n m, t+1}^{k}$. Then the total power is obtained as $\hat{E}_{n, t}^{l+1}=\sum_{m \in \omega_{n}} \hat{E}_{n m, t}^{l+1}$. After projecting into the feasible region (2), the projected total power $\hat{E}_{n, t}^{p r o j, l+1}$ is updated as

$$
\hat{E}_{n, t}^{p r o j, l+1}=\max \left\{\min \left\{\hat{E}_{n, t}^{l+1}, \bar{E}_{n, t}\right\}, \underline{E}_{n, t}\right\} .
$$

Then the trade $\hat{E}_{n m, t}^{l+1}$ to neighboring agent $m$ is updated as

$$
\hat{E}_{n m, t}^{l+1}=\hat{E}_{n m, t}^{l+1} \frac{\hat{E}_{n, t}^{p r o j, l+1}}{\hat{E}_{n, t}^{l+1}} .
$$

Finally, all agents send the new trade quantities $\hat{E}_{n m, t}^{l+1}$ to its neighboring agents, and check if the balance equation $\hat{E}_{n m, t}^{l+1}+\hat{E}_{m n, t}^{l+1}<\varepsilon$ is satisfied, where $\varepsilon$ is the allowed maximal violation; if not, repeat the processes (23)-(25) until all trades are balanced.

3) Extension to DC Power Flow Model: For simplicity, the power flow model and network constraints are not considered, but our P2P market mechanism can be extended to include the DC power flow model by adding a system operator (SO), who behaves as a single agent and helps to complete the calculation of power flows and voltage angles.

Let the power network consist of a set $\mathcal{N}$ of buses and a set $\mathcal{L}$ of lines. We consider the DC power flow model to characterize the line limits and the generation-load balance, which is widely used in market studies [37], [38]. Let $P_{i j, t}$ be the real power flow in the line connecting bus $i$ and $j, Y_{i j}$ is the susceptance of the line connecting bus $i$ and $j, \theta_{i, t}$ is the voltage angle at bus $i$, and $P_{i j}^{\min }, P_{i j}^{\max }$ are the line thermal limits. Without the loss of generality, let bus 1 be the slack bus, i.e., $\theta_{1, t}=0$. Then, the network constraints and power balance can be represented as follows,

$$
\begin{array}{r}
P_{i j}^{\min } \leq P_{i j, t}=Y_{i j}\left(\theta_{j, t}-\theta_{i, t}\right) \leq P_{i j}^{\max }, \forall(i, j) \in \mathcal{L} \\
\sum_{n \in \mathcal{N}_{i}} \hat{E}_{n, t}=\sum_{(i, j) \in \mathcal{L}} P_{i j, t}, \\
\forall i \in \mathcal{N}
\end{array}
$$

In transmission networks, the real power flows $P_{i j, t}$ are proportional to the difference of voltage angles between the two ends of the line. To avoid any damage to transmission lines, the power flows are bounded by thermal capacity related to the heat they can dissipate. Moreover, the power balance (26b) must be kept at each bus of the grid between line flows and power injections of agents connected to it. $\mathbf{P}_{t}=\left\{P_{i j, t},(i, j) \in \mathcal{L}\right\}$ and $\boldsymbol{\theta}_{t}=\left\{\theta_{i, t}, i \in \mathcal{N}\right\}$ are used to represent all the power flows in lines and voltage angles of buses at time step $t$.

The SO will be added as a single agent to complete the power flows and voltage angles calculation. The network constraints (26a)-(26b) can be converted into an optimization problem as

$$
\begin{aligned}
& \left\{\mathbf{P}_{t}, \boldsymbol{\theta}_{t}\right\}=\underset{\{\mathbf{P}, \boldsymbol{\theta}\}}{\operatorname{argmin}} \sum_{i \in \mathcal{N}}\left\|\sum_{n \in \mathcal{N}_{i}} \hat{E}_{n, t}-\sum_{(i, j) \in \mathcal{L}} P_{i j, t}\right\|_{2}^{2} \\
& \text { s.t. } \quad P_{i j}^{\min } \leq P_{i j, t}=Y_{i j}\left(\theta_{j}-\theta_{i}\right) \leq P_{i j}^{\max }(i, j) \in \mathcal{L}
\end{aligned}
$$

At each time step, the SO will collect the total power injection $\hat{E}_{n, t} n \in \Omega$ from all agents located at different buses, then determines the power flows $\mathbf{P}_{t}$ and voltage angles $\boldsymbol{\theta}_{t}$ by solving above problem (27).

Although extending the $\mathrm{P} 2 \mathrm{P}$ real-time market to include the DC power flow model requires the SO to solve the convex optimization problem (27), it is easy to solve, and the computational efficiency will not be highly reduced as demonstrated in the simulations.

Remark 3. If we consider the AC power flow, since the constraints have quadratic terms, the optimization problem for SO will be nonconvex and difficult to solve. To overcome this problem, we can linearize the constraints using LinDistFlow model [39], [40]. Then, the problem for SO will be convex, and also easy to solve. Compared with the optimization problem (27), the complexity and size of these two problems are close. The DC model and LinDistFlow model can be applied in different scenarios according to the network voltage level. The DC model is suitable for the HV network, while the LinDistFlow model is usually used in the LV network.

\section{MARKET ANALYSis}

In this section, we will first analyze the convergence performance for our proposed online algorithm, which is usually measured by the regret. Regret is the accumulated gap between the online solution and the best solution in hindsight [33], [41]-[43]. Under some standard assumptions, the sublinear regret upper bound for our algorithm is proved, which implies that social welfare will be maximized in the long run. Then, the four desirable properties of our proposed market mechanisms are analyzed. 


\section{A. Non-Stationary Regret Analysis}

The stationary regret is commonly adopted, which compares with the cost induced by the best and fixed decisions in hindsight:

$$
R(T)=\sum_{t=1}^{T}\left(\sum_{n \in \Omega} C_{n, t}\left(\hat{E}_{n, t}\right)\right)-\sum_{t=1}^{T}\left(\sum_{n \in \Omega} C_{n, t}\left(E_{n}^{*}\right)\right),
$$

where $E_{n}^{*}$ is the best and fixed decisions. Several sublinear regret upper bounds measured by stationary regret have been established in early works [33], [41], [42]. However, the stationary regret requires the decisions to remain unchanged throughout the period, which is not possible for the real-time electricity market, since the renewable power generation is usually changing with time, and the power balance will not be satisfied with fixed decisions. Recently, a new performance metric known as the non-stationary regret was proposed by [43]. The non-stationary regret compares the cumulative gap between the online decisions and the best solutions at each time step as defined below

$$
\tilde{R}(T)=\sum_{t=1}^{T}\left(\sum_{n \in \Omega} C_{n, t}\left(\hat{E}_{n, t}\right)\right)-\sum_{t=1}^{T}\left(\sum_{n \in \Omega} C_{n, t}\left(E_{n, t}^{*}\right)\right),
$$

where $E_{n, t}^{*}$ is the optimal solution of agent $n$ at time step $t$.

Before presenting the results, we introduce the some standard assumptions to derive the sublinear regret upper bound as presented later in Theorem 1 .

\section{Assumption 1.}

(a) Functions $C_{n, t}$ are convex with bounded subgradients, i.e., $\partial C_{n, t}\left(E_{n, t}\right) \leq G, \forall n \in \Omega$, with $G$ being a positive constant.

(b) The initial values are set to zero, i.e., $\lambda_{n m, 1}=0$ and $E_{n m, 1}=0, \forall(n, m) \in\left(\Omega, \omega_{n}\right)$.

(c) For any $t$, we assume the gap between the optimal and initial solutions are bounded, i.e., $\left(E_{n m, t}^{*}-E_{n m, 1}\right)^{2} \leq D_{1}$ and $\left(F_{n m, t}^{*}-F_{n m, 1}\right)^{2} \leq D_{2}, \forall(n, m) \in\left(\Omega, \omega_{n}\right)$, with $D_{1}$ and $\mathrm{D}_{2}$ being positive constants.

(d) Path variation is defined as the temporal change of the optimal solutions sequence, i.e., $V_{n m, T}=$ $\sum_{t=1}^{T}\left|E_{n m, t}^{*}-E_{n m, t+1}^{*}\right|$. We assume that the optimal solutions do not change dramatically, in other words, the path variation is bounded as $V_{n m, T} \leq P, \forall(n, m) \in$ $\left(\Omega, \omega_{n}\right)$, with $P$ being a positive constant called variation budget.

(e) The electricity prices do not change dramatically, or in mathematical sense, the price variation between two time steps is bounded as $\left|\lambda_{n m, t+1}-\lambda_{n m, t}\right| \leq \Lambda$, with $\Lambda$ being a positive constant.

In Assumption 1, (a)-(c) are generally required in the online optimization setting. For Assumption 1(d), [43] proved that if there is no restriction on the path variation, the non-stationary regret is linear in $T$ regardless of the strategies. Thus we assume that the path variation is bounded, which is also proposed in early works [43]-[45]. Finally, for Assumption $1(\mathrm{e})$, it is reasonable that the electricity price cannot be infinite in the electricity market background.
Bearing all above in mind, the following theorem establishes the regret bound for our online algorithm.

Theorem 1. OC-ADMM algorithm has the following sublinear non-stationary regret upper bound by setting $\rho=\sqrt{T}$ and $\eta=\sqrt{T}$

$$
\tilde{R}(T) \leq N(N-1)\left(\frac{D_{1}+D_{2}+\Lambda^{2}+G^{2}}{2}+L P\right) \sqrt{T},
$$

where $L=\max _{n \in \Omega} 2 *\left(\bar{E}_{n}-\underline{E}_{n}\right)$.

Proof. See Appendix A.

According to the above theorem, since the regret has $\mathcal{O}(\sqrt{T})$ upper bound, we have $\lim _{T \rightarrow \infty} \frac{\tilde{R}(T)}{T}=0$, which indicates that the gap of the social welfare (seen as a cost) between the optimal solutions are approaching to zero in the long run on time average. In the next subsection, we will analyze the desirable properties for a market-clearing mechanism based on Theorem 1 .

\section{B. Desirable Properties of Market Mechanism}

It is necessary and important to evaluate the quality of a market-clearing mechanism by checking the four desirable properties, which are market efficiency ${ }^{1}$, incentive compatibility $^{2}$, cost recovery ${ }^{3}$ and revenue adequacy ${ }^{4}$ [46]. Based on the Hurwicz theorem [46], no mechanism is capable of achieving all those properties at the same time.

1) Market efficiency: For our real-time P2P market, regret can be regarded as the cumulative total cost gap between our online solutions and optimal solutions. From Theorem 1, we have $\lim _{T \rightarrow \infty} \frac{\tilde{R}(T)}{T}=0$, which implies that the cost is minimized, in other words, social welfare and market efficiency are maximized in the long run.

2) Incentive compatibility: A market participant may gain profit by not trustfully offering in terms of price or quantity, but we assume that they are all non-strategic in this work.

3) Cost recovery: The individual profit for a producer at the time step $t$ is

$$
\sum_{m \in \omega_{n}} \lambda_{n m, t} E_{n m, t}-C_{n, t}\left(E_{n, t}\right)
$$

Since the quadratic cost function (5) is convex, monotonically increasing, and passing through the origin, the producer can always set $E_{n, t}=E_{n m, t}=0$ to avoid a negative profit. Thus the cost recovery is satisfied.

4) Revenue adequacy: From (12), the prices between two agents are identical, i.e., $\lambda_{n m, t}=\lambda_{m n, t}$, and after the projection, the power between agents is balanced, i.e., $\hat{E}_{n m, t}+$ $\hat{E}_{m n, t}=0$. Thus, the revenue adequacy is satisfied.

Summing up the above, our real-time P2P market mechanism satisfies most of the desirable properties.

\footnotetext{
${ }^{1}$ Market efficiency is maximized when outcomes maximize social welfare

${ }^{2} \mathrm{~A}$ mechanism is called incentive-compatible if every participant can maximize its objective just by acting according to its true preferences.

${ }^{3}$ Cost recovery implies that individual profit is non-negative.

${ }^{4}$ Revenue adequacy implies that there is no financial deficit in the market.
} 
TABLE I: Agents' initial characteristics of simple case study

\begin{tabular}{ccccc}
\hline \hline Agent & $a_{n, t}\left[\$ / \mathrm{kW}^{2}\right]$ & $b_{n, t}[\$ / \mathrm{kW}]$ & $\underline{E_{n}}[\mathrm{~kW}]$ & $\overline{E_{n}}[\mathrm{~kW}]$ \\
\hline \hline Generator & 0.0210 & 15.0413 & 0 & 4.9014 \\
User & 0.0144 & 6.4149 & -5.3252 & -2.4533 \\
Wind & 0.0100 & 5 & 1.3021 & 1.3021 \\
\hline \hline
\end{tabular}

TABLE II: Agents' changing characteristics of simple case study

\begin{tabular}{ccccccccccc}
\hline \hline Parameter & $\mathrm{t}=1$ & $\mathrm{t}=2$ & $\mathrm{t}=3$ & $\mathrm{t}=4$ & $\mathrm{t}=5$ & $\mathrm{t}=6$ & $\mathrm{t}=7$ & $\mathrm{t}=8$ & $\mathrm{t}=9$ & $\mathrm{t}=10$ \\
\hline \hline$a_{n, t}^{u}$ & 0.0144 & 0.0177 & 0.0119 & 0.0145 & 0.0171 & 0.0128 & 0.0166 & 0.0112 & 0.0196 & 0.0159 \\
$b_{n, t}^{u}$ & 6.9078 & 8.9760 & 7.4488 & 8.2316 & 8.7734 & 8.3985 & 5.8131 & 7.4918 & 6.7019 & 6.1191 \\
$E_{n, t}^{w}$ & 1.3021 & 1.3067 & 1.3067 & 1.2739 & 1.2985 & 1.2748 & 1.3012 & 1.2758 & 1.3018 & 1.3018 \\
\hline \hline
\end{tabular}

\section{Simulation Results}

As a basis for illustration and discussion, we first consider a simple case to show the trading prices and quantities. Then our algorithm is tested on a dataset that is taken every five minutes of wind power generation from 20 wind farms in Australia [47] for 30 days, to show the convergence and tracking performance. Finally, the computational efficiency is demonstrated by comparing with other methods in terms of computational time. To better show the performance, uniform distribution stochastic parameter settings are applied. We perform simulations using Matlab R2017b on a PC with $1.6 \mathrm{GHz}$ Intel Core 4 Duo CPU and 8 GB memory, and the convex optimization problem is solved by CVX Sedumi solver [48].

\section{A. Illustrative Case Study}

We first take a simple case with only one conventional generator, one wind generator, and one user, to show the trading process in terms of prices and quantities under the changing wind power generation and utility functions. The initial parameters of agents are summarized in Table I. We set the utility functions of user and wind power generation are changing with time, and the values are shown in Table. II. We also include the time-coupling constraints into the case, i.e., the ramp rate limit for conventional generator and total demand requirement for the user. The ramp rate limit is set to $0.5 \mathrm{~kW}$ per time step, and the total demand is set to $20 \mathrm{~kW}$ for 10 time steps.

The final transaction prices and quantities between agents in 10 time steps are shown in Fig. 1. The values are changing with time due to the varying user's characteristics and wind power generation. We give a comparison of the total cost between our online mechanism with the centralized mechanism, and the price between with pool-based mechanism. As seen in Fig. 2, the total cost of the online mechanism is higher than that of the centralized mechanism at some time steps, but the gap is very small. While for the price, in the poolbased market, the price is identical for all agents, while in our online mechanism, the prices are different between each pair of agents, and changing with time.
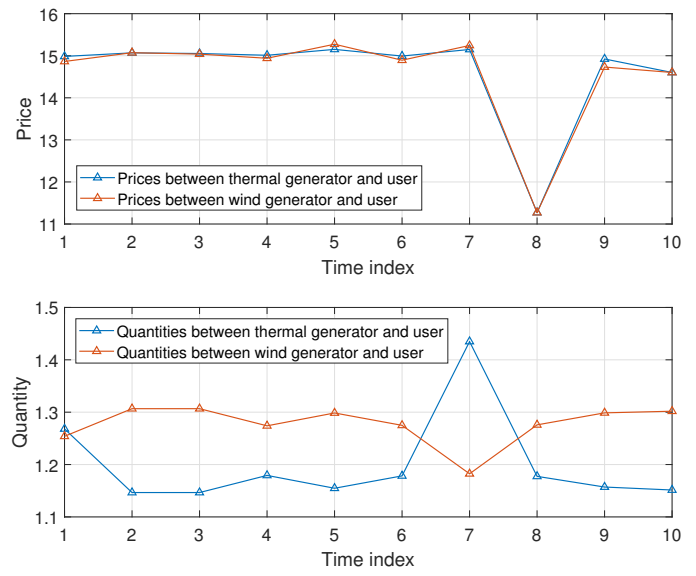

Fig. 1: The prices and quantities between agents.
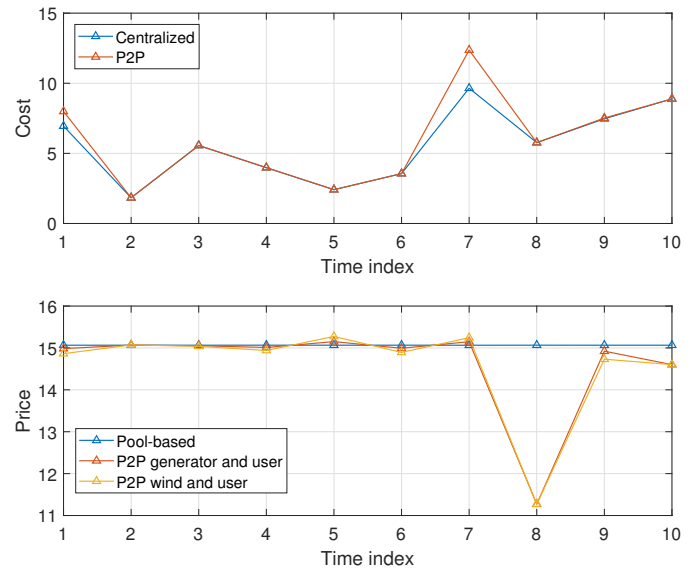

Fig. 2: The comparison of cost/price between P2P mechanism with centralized/pool-based mechanism. 


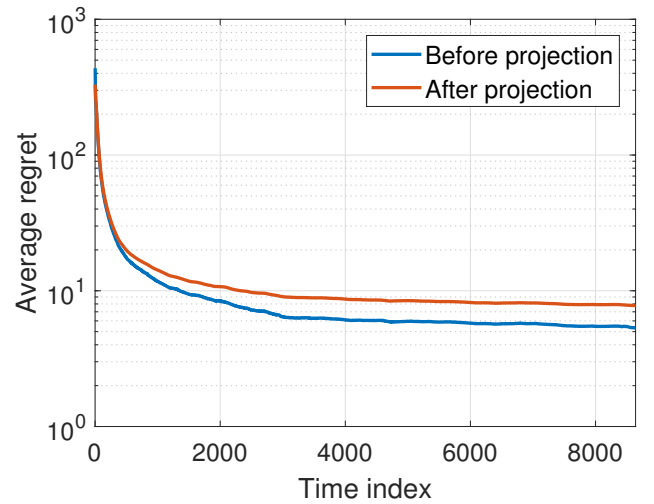

Fig. 3: Average regret $\tilde{R}(T) / T$ before and after projection.

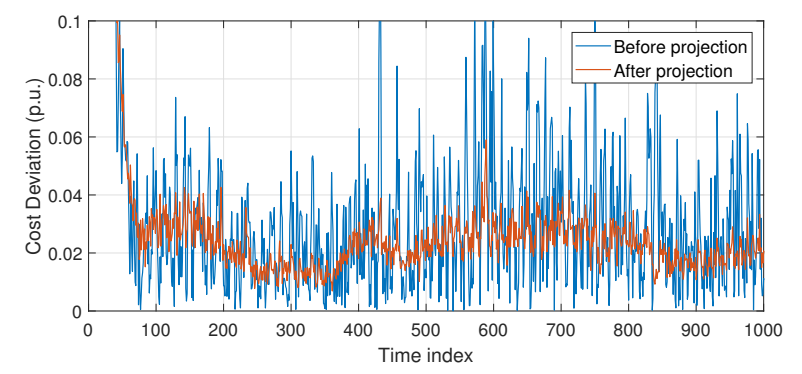

Fig. 4: The normalized cost deviation.

\section{B. Application to Real Data}

We build a large market composed of 20 conventional generators, 20 flexible residential users, and 20 wind power generators with the real wind generation data in Australia for 30 days. The convergence performance and tracking ability of our algorithm are tested.

1) Convergence Performance: Fig. 3 shows the convergence performance of our online algorithm before and after the projection. We can see $\tilde{R}(T) / T$ decreases quite fast both before and after projection, and the former one is lower. The reason for that is before projection, the balance constraint is relaxed and the solution is not feasible, thus the cost may be lower than that of the optimal solution. While after projection, since the solution is feasible, it will always exist a small positive gap from the optimal solution. It is shown that after about 2000 time steps, the average regret decreases much slower but still keeps going down.

2) Tracking Ability: Then we focus on the ability of tracking the optimal solution, a normalized index is proposed to measure the cost deviation

$$
\chi_{t}^{c} \triangleq \frac{\sum_{n \in \Omega}\left|C_{n, t}\left(E_{n, t}\right)-C_{n, t}\left(E_{n, t}^{*}\right)\right|}{\sum_{n \in \Omega}\left|C_{n, t}\left(E_{n, t}^{*}\right)\right|},
$$

The value of the index ranges from 0 to infinity, and the smaller value denotes better tracking ability. For better presentation, we only show the first 1000 time steps. Fig. 4 shows that the cost deviation keeps at a low level mostly under 0.08 before projection and 0.04 after projection, which implies that our algorithm can track the optimal solutions well.

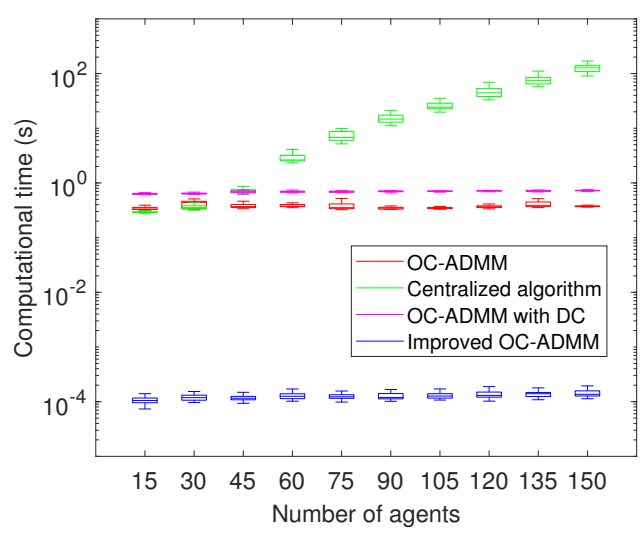

Fig. 5: The computational time of different methods.

\section{Computational Efficiency}

In this section, we will show the computational efficiency of our algorithm, measured by the computational time. Since the OC-ADMM is a fully decentralized algorithm, the computational time of one iteration is determined by the slowest agent. Simulations have been performed 30 times for the centralized algorithm (CVX Sedumi solver), OC-ADMM, OCADMM with DC, and improved OC-ADMM at a different scale of agents with different setups per iteration. As seen in Fig. 5, the OC-ADMM is quite efficient whose computational time is kept lower than 1s, and the improved OC-ADMM can further highly improve the performance to about $10^{-4} \mathrm{~s}$ with a little higher variance. For our proposed decentralized algorithms, the computational time almost remains unchanged, however, for the centralized algorithm, e.g., the interior point method, the computation time is exponentially increasing with the number of agents.

\section{Extension to DC Optimal Power Flow}

We first consider a small market with 10 agents on the IEEE 9-bus test system as shown in Fig. 6. The susceptance $Y$ and line capacity limit $C$ are set to 3 and 10 for all lines. Agent G1-G3 are conventional generators, agent U1-U4 are users, and agent R1-R3 are wind power generators. The power flows in different lines at the first 12 time steps are shown in Fig. 7. The results indicate that the power flows are also changing with time, and are always not larger than the maximum line capacity.

Then we run our online algorithm with the DC power flow model on the IEEE 57-bus test system, which is a larger system, to show the computational efficiency at a different scale of agents. Simulations have been performed 30 times for different numbers of agents (15 to 150) with different setups per iteration. As shown in Fig. 5, the computational time of OC-ADMM with DC also almost remains unchanged and below $1 \mathrm{sec}$. The time gap between OC-ADMM with and without DC is due to the time used by the SO, to calculate the power flows and phase angles, and the time is only affected by the network scale. We further give a simulation to show the impact of network scale on the computational time. 


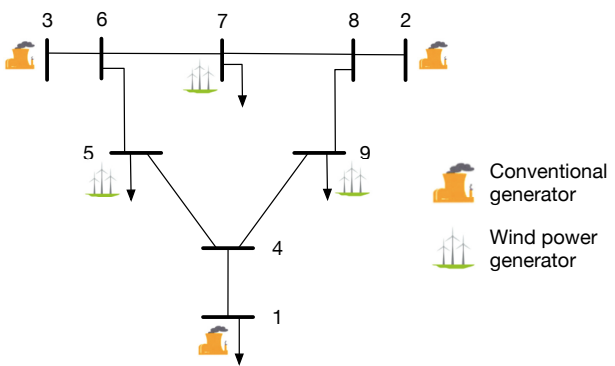

Fig. 6: IEEE 9-bus test system used for case study.

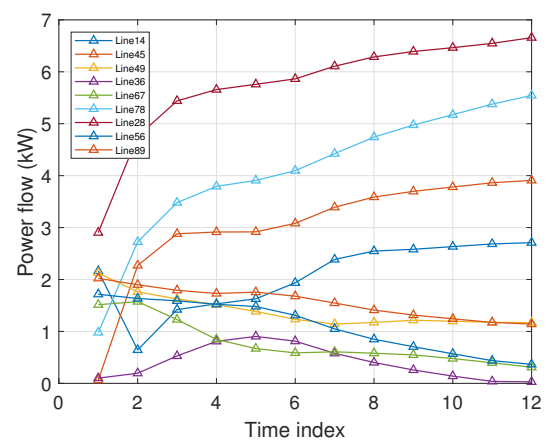

Fig. 7: Power flows in different lines at different time steps.

Simulations have been performed 30 times with 30 agents in each network. As shown in Fig. 8, the computational time will increase with the number of buses in the network, but the time is still much lower than the requirement for the real-time market (5 min).

\section{CONCLUSION}

P2P markets are considered as an evolution of future electricity markets driven by distributed energy resources and demand response management development. However, it is technically challenging to operate $\mathrm{P} 2 \mathrm{P}$ market mechanisms in real-time, since they usually involve a heavy computational burden, while the negotiation time before operation is quite short in real-time electricity markets. How to reduce the computational complexity of the P2P mechanism to be within a real-time architecture remains a challenge. To this end, we propose a novel OC-ADMM algorithm that incorporates the

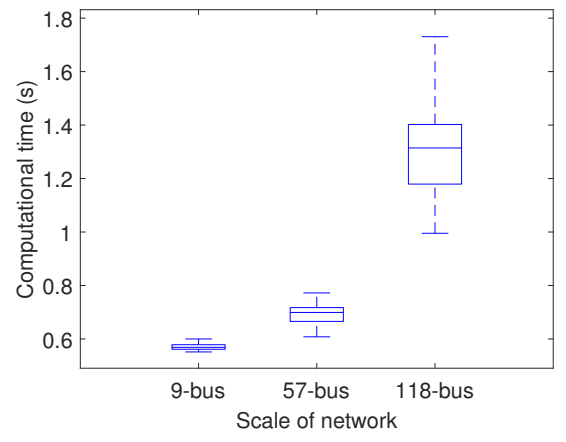

Fig. 8: Computational time as a function of network scale. online optimization approach to maximize social welfare in the long run. Since each agent only performs one iteration at every time step, the number of operations and communications among agents can be highly reduced, and the complexity of our approach is much less than others. Besides, some further improvements are made to increase performance and practicability. Finally, we give proof of the sublinear nonstationary regret upper bound for our algorithm, which indicates that social welfare will be maximized in the long run on time average. Simulation results show that our algorithm has good convergence performance, tracking ability, and high computational efficiency.

The main limitation of our approach is that the market mechanism has to be running in a synchronous manner. Each agent has to wait to receive all bid prices and quantities from neighboring agents, and the computational efficiency of the market is highly restricted by slow and low-reliability agents. To overcome this problem, we are working on designing an asynchronous mechanism to make it more practical and applicable in the real world.

\section{APPENDIX A}

\section{PROOF OF THE SUblineAr REgRET UPPER BOUND}

Let $\frac{\partial C_{n, t}\left(E_{n, t}\right)}{\partial E_{n m, t}}$ be the gradient of $C_{n, t}\left(E_{n}\right)$ at $E_{n m, t}$. Since $\mathbf{E}_{n, t}$ minimizes (11), combining (12), we have

$$
\begin{aligned}
& \frac{\partial C_{n, t}\left(E_{n, t}\right)}{\partial E_{n m, t}}=\lambda_{n m, t}+\rho\left(F_{n m, t-1}-F_{n m, t}\right) \\
& +\eta\left(E_{n m, t-1}-E_{n m, t}\right), \forall m \in \omega_{n} .
\end{aligned}
$$

Since $C_{n, t}$ is a convex function and its subgradient at $E_{n m, t}$ is given in (33), for optimal solution $E_{n, t}^{*}$ we have

$$
\begin{aligned}
& C_{n, t}\left(E_{n, t}\right)-C_{n, t}\left(E_{n, t}^{*}\right) \\
& \leq \sum_{m \in \omega_{n}} \frac{\partial C_{n, t}\left(E_{n, t}\right)}{\partial E_{n m, t}}\left(E_{n m, t}-E_{n m, t}^{*}\right) \\
&= \sum_{m \in \omega_{n}} \lambda_{n m, t}\left(E_{n m, t}-F_{n m, t}^{*}\right) \\
&+\sum_{m \in \omega_{n}} \rho\left(F_{n m, t-1}-F_{n m, t}\right)\left(E_{n m, t}-F_{n m, t}^{*}\right) \\
&+\sum_{m \in \omega_{n}} \eta\left(E_{n m, t-1}-E_{n m, t}\right)\left(E_{n m, t}-E_{n m, t}^{*}\right) \\
&= \sum_{m \in \omega_{n}} \lambda_{n m, t}\left(E_{n m, t}-F_{n m, t}^{*}\right) \\
&+\sum_{m \in \omega_{n}} \frac{\rho}{2}\left[\left(F_{n m, t-1}-F_{n m, t}^{*}\right)^{2}-\left(F_{n m, t}-F_{n m, t}^{*}\right)^{2}\right. \\
&\left.+\left(E_{n m, t}-F_{n m, t}\right)^{2}-\left(E_{n m, t}-F_{n m, t-1}\right)^{2}\right] \\
&+\frac{\eta}{2}\left[\left(E_{n m, t}^{*}-E_{n m, t-1}\right)^{2}-\left(E_{n m, t}^{*}-E_{n m, t}\right)^{2}\right. \\
&\left.-\left(E_{n m, t}-E_{n m, t-1}\right)^{2}\right] .
\end{aligned}
$$

According to the Fenchel-Young's inequality [49], we have

$C_{n, t}\left(\hat{E}_{n, t}\right)-C_{n, t}\left(E_{n, t}\right)$

$\leq \sum_{m \in \omega_{n}} \frac{\partial C_{n, t}\left(\hat{E}_{n, t}\right)}{\partial \hat{E}_{n m, t}}\left(\hat{E}_{n m, t}-E_{n m, t}\right)$ 


$$
\begin{aligned}
& \leq \sum_{m \in \omega_{n}} \frac{1}{2 \rho}\left(\frac{\partial C_{n, t}\left(\hat{E}_{n, t}\right)}{\partial \hat{E}_{n m, t}}\right)^{2}+\frac{\rho}{2}\left(\hat{E}_{n m, t}-E_{n m, t}\right)^{2} \\
& \leq \sum_{m \in \omega_{n}} \frac{1}{2 \rho}\left(\frac{\partial C_{n, t}\left(\hat{E}_{n, t}\right)}{\partial \hat{E}_{n m, t}}\right)^{2}+\frac{\rho}{2}\left(F_{n m, t}-E_{n m, t}\right)^{2} \\
& \leq \sum_{m \in \omega_{n}} \frac{1}{2 \rho}\left(\frac{\partial C_{n, t}\left(\hat{E}_{n, t}\right)}{\partial \hat{E}_{n m, t}}\right)^{2}+\frac{\left(\lambda_{n m, t-1}-\lambda_{n m, t}\right)^{2}}{2 \rho}
\end{aligned}
$$

For the penultimate inequality, because $\hat{E}_{n m, t}$ begins updating from $F_{n m, t}$, if the projection update finishes at the first time, which also means both agents do not touch the bound, the term $\left(\hat{E}_{n m, t}-E_{n m, t}\right)^{2}$ reaches the maximal value $\left(F_{n m, t}-\right.$ $\left.E_{n m, t}\right)^{2}$; The worst case is that the final trade reaches at the initial value $E_{n m, t}$ or $-E_{m n, t}$, which means at the beginning, one of the agents $n$ or $m$ has reached the bound, then the term $\left(\hat{E}_{n m, t}-E_{n m, t}\right)^{2}$ reaches the minimal value zero.

Combining (34)-(35), we have

$$
\begin{aligned}
& C_{n, t}\left(\hat{E}_{n, t}\right)-C_{n, t}\left(E_{n, t}^{*}\right) \\
& \leq \sum_{m \in \omega_{n}} \lambda_{n m, t}\left(E_{n m, t}-F_{n m, t}^{*}\right)+\frac{\rho}{2}\left(E_{n m, t}-F_{n m, t}\right)^{2} \\
& \quad+\sum_{m \in \omega_{n}} \frac{\rho}{2}\left[\left(F_{n m, t-1}-F_{n m, t}^{*}\right)^{2}-\left(F_{n m, t}-F_{n m, t}^{*}\right)^{2}\right] \\
& \quad+\sum_{m \in \omega_{n}} \frac{\eta}{2}\left[\left(E_{n m, t}^{*}-E_{n m, t-1}\right)^{2}-\left(E_{n m, t}^{*}-E_{n m, t}\right)^{2}\right] \\
& \quad+\sum_{m \in \omega_{n}} \frac{1}{2 \rho}\left(\frac{\partial C_{n, t}\left(\hat{E}_{n, t}\right)}{\partial \hat{E}_{n m, t}}\right)^{2}+\frac{\left(\lambda_{n m, t-1}-\lambda_{n m, t}\right)^{2}}{2 \rho}
\end{aligned}
$$

We notice that for the second term

$$
\begin{aligned}
( & \left.F_{n m, t-1}-F_{n m, t}^{*}\right)^{2}-\left(F_{n m, t}-F_{n m, t}^{*}\right)^{2} \\
= & \left(F_{n m, t-1}-F_{n m, t-1}^{*}\right)^{2}-\left(F_{n m, t}-F_{n m, t}^{*}\right)^{2} \\
& +\left(F_{n m, t-1}-F_{n m, t}^{*}\right)^{2}-\left(F_{n m, t-1}-F_{n m, t-1}^{*}\right)^{2} \\
\leq & \left(F_{n m, t-1}-F_{n m, t-1}^{*}\right)^{2}-\left(F_{n m, t}-F_{n m, t}^{*}\right)^{2} \\
& +\left(2 F_{n m, t-1}-F_{n m, t-1}^{*}-F_{n m, t}^{*}\right)\left(F_{n m, t-1}^{*}-F_{n m, t}^{*}\right) \\
\leq & \left(F_{n m, t-1}-F_{n m, t-1}^{*}\right)^{2}-\left(F_{n m, t}-F_{n m, t}^{*}\right)^{2} \\
& +L\left|E_{n m, t-1}^{*}-E_{n m, t}^{*}\right| .
\end{aligned}
$$

Similarly, for the last term

$$
\begin{aligned}
& \left(E_{n m, t}^{*}-E_{n m, t-1}\right)^{2}-\left(E_{n m, t}^{*}-E_{n m, t}\right)^{2} \\
& \leq\left(E_{n m, t-1}-E_{n m, t-1}^{*}\right)^{2}-\left(E_{n m, t}-E_{n m, t}^{*}\right)^{2} \\
& \quad+L\left|E_{n m, t-1}^{*}-E_{n m, t}^{*}\right| .
\end{aligned}
$$

For the first term, using $E_{n m, t}-F_{n m, t}=\frac{\lambda_{n m, t-1}-\lambda_{n m, t}}{\rho}$, $F_{n m, t}^{*}+F_{m n, t}^{*}=0, \lambda_{n m, t}=\lambda_{m n, t}$ and summing up for all $n \in \Omega$ yields

$$
\begin{aligned}
& \sum_{n \in \Omega} \sum_{m \in \omega_{n}} \lambda_{n m, t}\left(E_{n m, t}-F_{n m, t}^{*}\right)+\frac{\rho}{2}\left(E_{n m, t}-F_{n m, t}\right)^{2} \\
& =\frac{1}{2} \sum_{\forall(n, m) \in\left(\Omega, \omega_{n}\right)} \lambda_{n m, t}\left[E_{n m, t}+E_{m n, t}-\left(F_{n m, t}^{*}+F_{m n, t}^{*}\right]\right] \\
& \quad+\frac{\rho}{2}\left(E_{n m, t}-F_{n m, t}\right)^{2}+\frac{\rho}{2}\left(E_{m n, t}-F_{m n, t}\right)^{2}
\end{aligned}
$$

$$
\begin{aligned}
= & \frac{1}{2} \sum_{\forall(n, m) \in\left(\Omega, \omega_{n}\right)} 2 \lambda_{n m, t}\left(E_{n m, t}-F_{n m, t}\right) \\
& +\frac{1}{2 \rho}\left(\lambda_{n m, t-1}-\lambda_{n m, t}\right)^{2}+\frac{1}{2 \rho}\left(\lambda_{m n, t-1}-\lambda_{m n, t}\right)^{2} \\
= & \sum_{\forall(n, m) \in\left(\Omega, \omega_{n}\right)} \frac{1}{2 \rho}\left(\lambda_{n m, t-1}^{2}-\lambda_{n m, t}^{2}\right) .
\end{aligned}
$$

Combining (36)-(39) and based on Assumption 1, $\tilde{R}(T)$ is bounded as follows

$$
\begin{aligned}
\sum_{t=1}^{T} & \left(\sum_{n \in \Omega} C_{n, t}\left(\hat{E}_{n, t}\right)\right)-\sum_{t=1}^{T}\left(\sum_{n \in \Omega} C_{n, t}\left(E_{n, t}^{*}\right)\right) \\
\leq & \sum_{\forall(n, m) \in\left(\Omega, \omega_{n}\right)} \frac{1}{2 \rho}\left(\lambda_{n m, 1}^{2}-\lambda_{n m, T}^{2}\right) \\
& +\sum_{n \in \Omega} \sum_{m \in \omega_{n}} \frac{\rho}{2}\left[\left(F_{n m, 1}-F_{n m, 1}^{*}\right)^{2}-\left(F_{n m, T}-F_{n m, T}^{*}\right)^{2}\right] \\
& +\sum_{n \in \Omega} \sum_{m \in \omega_{n}} \frac{\eta}{2}\left[\left(E_{n m, 1}-E_{n m, 1}^{*}\right)^{2}-\left(E_{n m, T}-E_{n m, T}^{*}\right)^{2}\right] \\
& +\sum_{t=1}^{T} \sum_{n \in \Omega} \sum_{m \in \omega_{n}} \frac{(\rho+\eta) L}{2}\left|E_{n m, t}^{*}-E_{n m, t}^{*}\right| \\
& +\sum_{t=1}^{T} \sum_{n \in \Omega} \sum_{m \in \omega_{n}} \frac{1}{2 \rho}\left(\frac{\partial C_{n, t}\left(\hat{E}_{n, t}\right)}{\partial \hat{E}_{n m, t}}\right)^{2} \\
& +\sum_{t=1}^{T} \sum_{n \in \Omega} \sum_{m \in \omega_{n}} \frac{\left(\lambda_{n m, t-1}-\lambda_{n m, t}\right)^{2}}{2 \rho} \\
\leq & N(N-1)\left(\frac{\rho D_{2}}{2}+\frac{\eta D_{1}}{2}+\frac{(\rho+\eta) L P}{2}+\frac{T G^{2}}{2 \rho}+\frac{T \Lambda^{2}}{2 \rho}\right) .
\end{aligned}
$$

Setting $\rho=\sqrt{T}$ and $\eta=\sqrt{T}$ yields sublinear regret $\tilde{R}(T)$.

\section{REFERENCES}

[1] J. Hu, R. Harmsen, W. Crijns-Graus, E. Worrell, and M. van den Broek, "Identifying barriers to large-scale integration of variable renewable electricity into the electricity market: A literature review of market design," Renewable and Sustainable Energy Reviews, vol. 81, pp. 21812195, 2018.

[2] Y. Parag and B. K. Sovacool, "Electricity market design for the prosumer era," Nature energy, vol. 1, no. 4, pp. 1-6, 2016.

[3] T. Baroche, P. Pinson, R. L. G. Latimier, and H. B. Ahmed, "Exogenous cost allocation in peer-to-peer electricity markets," IEEE Transactions on Power Systems, vol. 34, no. 4, pp. 2553-2564, 2019.

[4] M. Khorasany, Y. Mishra, and G. Ledwich, "A decentralized bilateral energy trading system for peer-to-peer electricity markets," IEEE Transactions on Industrial Electronics, vol. 67, no. 6, pp. 4646-4657, 2020.

[5] T. Sousa, T. Soares, P. Pinson, F. Moret, T. Baroche, and E. Sorin, "Peer-to-peer and community-based markets: A comprehensive review," Renewable and Sustainable Energy Reviews, vol. 104, pp. 367-378, 2019.

[6] F. Moret, T. Baroche, E. Sorin, and P. Pinson, "Negotiation algorithms for peer-to-peer electricity markets: Computational properties," in 2018 Power Systems Computation Conference (PSCC), pp. 1-7, IEEE, 2018.

[7] R. L. G. Latimier, T. Baroche, and H. B. Ahmed, "Mitigation of communication costs in peer-to-peer electricity markets," in 2019 IEEE Milan PowerTech, pp. 1-6, IEEE, 2019.

[8] F. Moret and P. Pinson, "Energy collectives: a community and fairness based approach to future electricity markets," IEEE Transactions on Power Systems, vol. 34, no. 5, pp. 3994-4004, 2019.

[9] E. Sorin, L. Bobo, and P. Pinson, "Consensus-based approach to peer-topeer electricity markets with product differentiation," IEEE Transactions on Power Systems, vol. 34, no. 2, pp. 994-1004, 2018. 
[10] A. Lüth, J. M. Zepter, P. C. del Granado, and R. Egging, "Local electricity market designs for peer-to-peer trading: The role of battery flexibility," Applied energy, vol. 229, pp. 1233-1243, 2018.

[11] J. M. Zepter, A. Lüth, P. C. del Granado, and R. Egging, "Prosumer integration in wholesale electricity markets: Synergies of peer-to-peer trade and residential storage," Energy and Buildings, vol. 184, pp. 163176, 2019.

[12] Q. Wang, C. Zhang, Y. Ding, G. Xydis, J. Wang, and J. Østergaard, "Review of real-time electricity markets for integrating distributed energy resources and demand response," Applied Energy, vol. 138, pp. 695-706, 2015.

[13] T. Namerikawa, N. Okubo, R. Sato, Y. Okawa, and M. Ono, "Realtime pricing mechanism for electricity market with built-in incentive for participation," IEEE Transactions on Smart Grid, vol. 6, no. 6, pp. 2714 $2724,2015$.

[14] A. W. Berger and F. C. Schweppe, "Real time pricing to assist in load frequency control," IEEE Transactions on Power Systems, vol. 4, no. 3, pp. 920-926, 1989.

[15] P. Samadi, H. Mohsenian-Rad, R. Schober, and V. W. Wong, "Advanced demand side management for the future smart grid using mechanism design," IEEE Transactions on Smart Grid, vol. 3, no. 3, pp. 1170$1180,2012$.

[16] S. Pineda and A. J. Conejo, "Using electricity options to hedge against financial risks of power producers," Journal of Modern Power Systems and Clean Energy, vol. 1, no. 2, pp. 101-109, 2013.

[17] V. C. Gungor, D. Sahin, T. Kocak, S. Ergut, C. Buccella, C. Cecati, and G. P. Hancke, "Smart grid technologies: Communication technologies and standards," IEEE transactions on Industrial informatics, vol. 7, no. 4, pp. 529-539, 2011

[18] T. Morstyn, A. Teytelboym, and M. D. McCulloch, "Bilateral contract networks for peer-to-peer energy trading," IEEE Transactions on Smart Grid, vol. 10, no. 2, pp. 2026-2035, 2018.

[19] Z. Zhang, R. Li, and F. Li, "A novel peer-to-peer local electricity market for joint trading of energy and uncertainty," IEEE Transactions on Smart Grid, vol. 11, no. 2, pp. 1205-1215, 2019

[20] E. Münsing, J. Mather, and S. Moura, "Blockchains for decentralized optimization of energy resources in microgrid networks," in 2017 IEEE conference on control technology and applications (CCTA), pp. 2164 2171, IEEE, 2017.

[21] S. Wang, A. F. Taha, J. Wang, K. Kvaternik, and A. Hahn, "Energy crowdsourcing and peer-to-peer energy trading in blockchain-enabled smart grids," IEEE Transactions on Systems, Man, and Cybernetics: Systems, vol. 49, no. 8, pp. 1612-1623, 2019.

[22] Z. Guo, P. Pinson, S. Chen, Q. Yang, and Z. Yang, "Chance-constrained peer-to-peer joint energy and reserve market considering renewable generation uncertainty," IEEE Transactions on Smart Grid, vol. 12, no. 1, pp. $798-809,2020$.

[23] D. H. Nguyen, "Optimal solution analysis and decentralized mechanisms for peer-to-peer energy markets," IEEE Transactions on Power Systems, 2020.

[24] E. Hazan, "Introduction to online convex optimization," arXiv preprint arXiv: 1909.05207, 2019.

[25] E. C. Hall and R. M. Willett, "Online convex optimization in dynamic environments," IEEE Journal of Selected Topics in Signal Processing, vol. 9, no. 4, pp. 647-662, 2015.

[26] S. Shalev-Shwartz et al., "Online learning and online convex optimization," Foundations and trends in Machine Learning, vol. 4, no. 2 , pp. 107-194, 2011

[27] T. Chen and G. B. Giannakis, "Bandit convex optimization for scalable and dynamic iot management," IEEE Internet of Things Journal, vol. 6 , no. 1, pp. 1276-1286, 2018.

[28] T. Chen, Q. Ling, and G. B. Giannakis, "An online convex optimization approach to proactive network resource allocation," IEEE Transactions on Signal Processing, vol. 65, no. 24, pp. 6350-6364, 2017.

[29] S.-J. Kim and G. B. Giannakis, "An online convex optimization approach to real-time energy pricing for demand response," IEEE Transactions on Smart Grid, vol. 8, no. 6, pp. 2784-2793, 2016.

[30] A. Lesage-Landry and J. A. Taylor, "Setpoint tracking with partially observed loads," IEEE Transactions on Power Systems, vol. 33, no. 5, pp. 5615-5627, 2018.

[31] W.-J. Ma, J. Wang, V. Gupta, and C. Chen, "Distributed energy management for networked microgrids using online admm with regret," IEEE Transactions on Smart Grid, vol. 9, no. 2, pp. 847-856, 2016.

[32] R. H. Day, "Rational choice and economic behavior," Theory and decision, vol. 1, no. 3, pp. 229-251, 1971.

[33] H. Wang and A. Banerjee, "Online alternating direction method (longer version)," arXiv preprint arXiv:1306.3721, 2013.
[34] H.-F. Xu, Q. Ling, and A. Ribeiro, "Online learning over a decentralized network through admm," Journal of the Operations Research Society of China, vol. 3, no. 4, pp. 537-562, 2015.

[35] S. Boyd, N. Parikh, E. Chu, B. Peleato, and J. Eckstein, "Distributed optimization and statistical learning via the alternating direction method of multipliers," Foundations and Trends in Machine Learning, vol. 3, no. 1, pp. 1-122, 2010.

[36] S. Boyd, S. P. Boyd, and L. Vandenberghe, Convex optimization. Cambridge university press, 2010.

[37] N. Gatsis and G. B. Giannakis, "Decomposition Algorithms for Market Clearing With Large-Scale Demand Response," IEEE Transactions on Smart Grid, vol. 4, no. 4, pp. 1976-1987, 2013.

[38] A. Kargarian, J. Mohammadi, J. Guo, S. Chakrabarti, M. Barati, G. Hug, S. Kar, and R. Baldick, "Toward Distributed/Decentralized DC Optimal Power Flow Implementation in Future Electric Power Systems," IEEE Transactions on Smart Grid, vol. 9, no. 4, pp. 2574-2594, 2018.

[39] M. E. Baran and F. F. Wu, "Optimal capacitor placement on radial distribution systems," IEEE Transactions on power Delivery, vol. 4. no. 1, pp. 725-734, 1989 .

[40] Z. Wang, B. Chen, J. Wang, M. M. Begovic, and C. Chen, "Coordinated energy management of networked microgrids in distribution systems," IEEE Transactions on Smart Grid, vol. 6, no. 1, pp. 45-53, 2014.

[41] M. Zinkevich, "Online convex programming and generalized infinitesimal gradient ascent," in Proceedings of the 20th international conference on machine learning (icml-03), pp. 928-936, 2003.

[42] E. Hazan, A. Agarwal, and S. Kale, "Logarithmic regret algorithms for online convex optimization," Machine Learning, vol. 69, no. 2-3, pp. 169-192, 2007.

[43] O. Besbes, Y. Gur, and A. Zeevi, "Non-stationary stochastic optimization," Operations research, vol. 63, no. 5, pp. 1227-1244, 2015.

[44] X. Gao, X. Li, and S. Zhang, "Online learning with non-convex losses and non-stationary regret," in International Conference on Artificial Intelligence and Statistics, pp. 235-243, 2018.

[45] S. Shahrampour and A. Jadbabaie, "Distributed online optimization in dynamic environments using mirror descent," IEEE Transactions on Automatic Control, vol. 63, no. 3, pp. 714-725, 2017.

[46] L. Hurwicz, "On informationally decentralized systems," Decision and organization: A volume in Honor of J. Marschak, 1972.

[47] J. Dowell and P. Pinson, "Very-short-term probabilistic wind power forecasts by sparse vector autoregression," IEEE Transactions on Smart Grid, vol. 7, no. 2, pp. 763-770, 2015.

[48] M. Grant, S. Boyd, and Y. Ye, "cvx users' guide," 2009.

[49] R. T. Rockafellar, Convex Analysis, vol. 36. Princeton University Press, 2015.

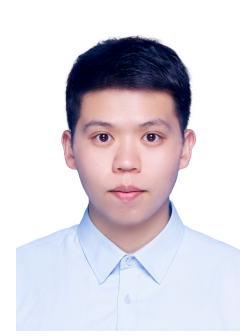

Zhenwei Guo received the B.S. degree in automation from Zhejiang University, Hangzhou, China, in 2016. Currently, he is pursuing the Ph.D. degree in the College of Control Science and Engineering, at Zhejiang University. He is a member of the Group of Networked Sensing and Control in the State Key Laboratory of Industrial Control Technology at Zhejiang University. His research interests include demand side management, distributed optimization and electricity market in smart grid.

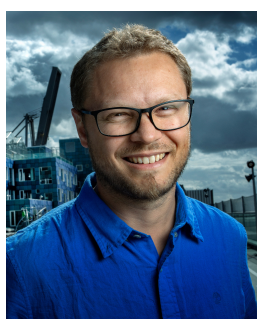

Pierre Pinson (SM'13, F'20) received the M.Sc. degree in applied mathematics from the National Institute for Applied Sciences, Toulouse, France, and the $\mathrm{Ph} . \mathrm{D}$. degree in energetics from Ecole des Mines de Paris, Paris, France. He is a Professor with the Centre for Electric Power and Energy, Department of Electrical Engineering, Technical University of Denmark, Lyngby, Denmark, also heading a group focusing on energy analytics and markets. His research interests include forecasting, uncertainty estimation, optimization under uncertainty, decision sciences, and renewable energies. He is the Editor-in-Chief for the International Journal of Forecasting. 


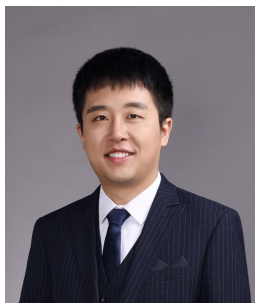

Shibo Chen (M'17) received the B.Eng. degree in electronic engineering from University of Science and Technology of China (USTC), Hefei, China, in 2011 and the Ph.D. degree in electronic and computer engineering from the Hong Kong University of Science and Technology (HKUST), Kowloon, Hong Kong, in 2017. He was a Postdoctoral Fellow with HKUST before joining the Department of Mechanical and Energy Engineering, Southern University of Science and Technology (SUSTech), Shenzhen, China in 2019 as a Research Assistant Professor. His current research interests include smart grid, optimization theory and game theory.

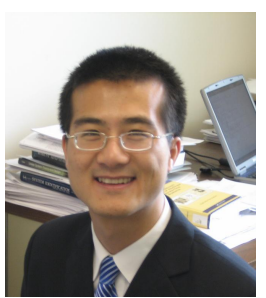

Qinmin Yang received the Bachelor's degree in Electrical Engineering from Civil Aviation University of China, Tianjin, China in 2001, the Master of Science Degree in Control Science and Engineering from Institute of Automation, Chinese Academy of Sciences, Beijing, China in 2004, and the Ph.D. degree in Electrical Engineering from the University of Missouri-Rolla, MO USA, in 2007. From 2007 to 2008, he was a Post-doctoral Research Associate at University of Missouri-Rolla. From 2008 to 2009, he was a system engineer with Caterpillar Inc. From 2009 to 2010, he was a Post-doctoral Research Associate at University of Connecticut. Since 2010, he has been with the State Key Laboratory of Industrial Control Technology, the College of Control Science and Engineering, Zhejiang University, China, where he is currently a professor. He has also held visiting positions in University of Toronto and Lehigh University. He has been serving as an Associate Editor for IEEE Transactions on Systems, Man, and Cybernetics: Systems, Transactions of the Institute of Measurement and Control, and Automatica Sinica. His research interests include intelligent control, renewable energy systems, smart grid, and industrial big data.

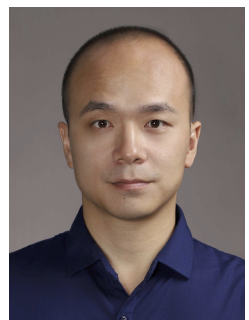

Zaiyue Yang (M'10) received the B.S. and M.S degrees from the Department of Automation, University of Science and Technology of China, Hefei, China, in 2001 and 2004, respectively, and the Ph.D. degree from the Department of Mechanical Engineering, University of Hong Kong, in 2008. He was a Postdoctoral Fellow and Research Associate with the Department of Applied Mathematics, Hong Kong Polytechnic University, before joining the College of Control Science and Engineering, Zhejiang University, Hangzhou, China, in 2010. Then, he joined the Department of Mechanical and Energy Engineering, Southern University of Science and Technology, Shenzhen, China, in 2017. He is currently a Professor there. His current research interests include smart grid, signal processing and control theory. Prof. Yang is an associate editor for the IEEE Transactions on Industrial Informatics. 